\title{
The Methylene Tetrahydrofolate Reductase (MTHFR) isoform challenge. High doses of folic acid are not a suitable option compared to 5 Methyltetrahydrofolate treatment
}

\author{
Edouard Servy ${ }^{1 *}$ and Yves Menezo ${ }^{2}$ \\ ${ }^{1}$ Servy Massey Fertility Institute, 812 Chafee Ave, Augusta Ga, USA \\ ${ }^{2}$ London Fertility Associates, Harley St, London UK and Laboratoire CLEMENT, avenue d'Eylau, Paris, France
}

\begin{abstract}
Methylene Tetrahydofolate Reductase (MTHFR) is an enzyme participating to the "folic acid cycle" which is linked to the one carbon cycle (1-CC). These combined metabolic cycles are involved in methylation, a major biochemical process. Methylation is performed on lipids, proteins and DNA. DNA methylation is involved in major cellular processes such as gene transcription, genomic imprinting and epigenesis. MTHFR have several genetic isoforms: the most common are C677T and A1298C, both of them are frequent and negatively affect the methylation process, as they impair the formation of the metabolically active folate $5 \mathrm{MTHF}$ (Methylene tetrahydrofolate). This biochemical deficiency alters strongly fertility through a deleterious impact on gamete quality. We discuss here the necessity of testing the MTHFR in case of long lasting impaired fertility and repeat miscarriages. We present also our preliminary data associated with literature data indicating that high doses of Folic acid, a synthetic compound, are not a reasonable option. These patients must be treated with $5 \mathrm{MTHF}$, associated with a support of the $1-\mathrm{CC}$ a in order to back up the methylation process.
\end{abstract}

\section{Introduction}

Methylene Tetrahydofolate Reductase (MTHFR) affects the distribution of folate and maintains a delicate balance between folate for nucleotide synthesis and those for methionine synthesis MTHFR isoforms are well known genetic variants linked to the one-carboncycle (1-CC), also known as methionine and methylation cycle (Figure 1). The gene for MTHFR enzyme is one of the 20,000 genes. It is located on chromosome 1 in our DNA. At least 35 different types of the mutation have been researched. The most common being studied are the C677T and A1298C. There are various degrees of gene mutations. DNA analysis of the MTHFR gene is usually performed by PCR amplification followed by restriction analysis. The diagnostic sensitivity is $>99 \%$ for both mutations [1].

MTHFR C677C $=$ normal MTHFR gene

MTHFR C677T $=$ heterozygous mutation (one mutation)

MTHFR T677T = homozygous mutation (two mutations)

MTHFR A1298A = normal MTHFR gene

MTHFR A1298C = heterozygous mutation (one mutation)

MTHFR C1298C = homozygous mutation (two mutations)

MTHFR C677T + MTHFR A1298C = compound heterozygous mutation

If an individual carries an MTHFR isoform he may produce $17 \%$ (MTHFR C1298C) to 75\% (MTHFR T677T) less methyl folate than someone without the mutation. MTHFR is necessary for the conversion of homocysteine to methionine. Heterozygous mutation elicits rather minor influence on gene activity and health. When dealing with 677T polymorphism, homozygotes deliver only $20 \%$ or less of the enzymatic activity. For 1298C homozygotes, the enzymatic activity may only reach $68 \%$ of the normal. Compound heterozygous mutation can also affect substantially the gene activity [2-4].

\section{Epidemiology}

The table 1 below shows the results of numerous studies conducted worldwide looking at the C677T mutation showing the rates of carrier and affected populations according to the ethnicity [3].

It is estimated that a third of the world population is a carrier of MTHFR mutation, while the percent affected is less than $10 \%$ of the population [5].

\section{Methylation, a major biochemical process in gameto- genesis}

Methylation adds methyl groups covalently to several molecules: lipids, proteins and DNA. In the reproduction field, the most important

Correspondence to: Edouard Servy, Servy Massey Fertility Institute, 812 Chafee Ave, Augusta Ga, USA; E-mail: eservy@yahoo.com

Key words: MTHFR isoforms, folic acid, 5 Methyl Tetra Hydrofolate, methylation, infertility, recurrent miscarriages

Received: November 03, 2017; Accepted: November 20, 2017; Published: November 23, 2017 


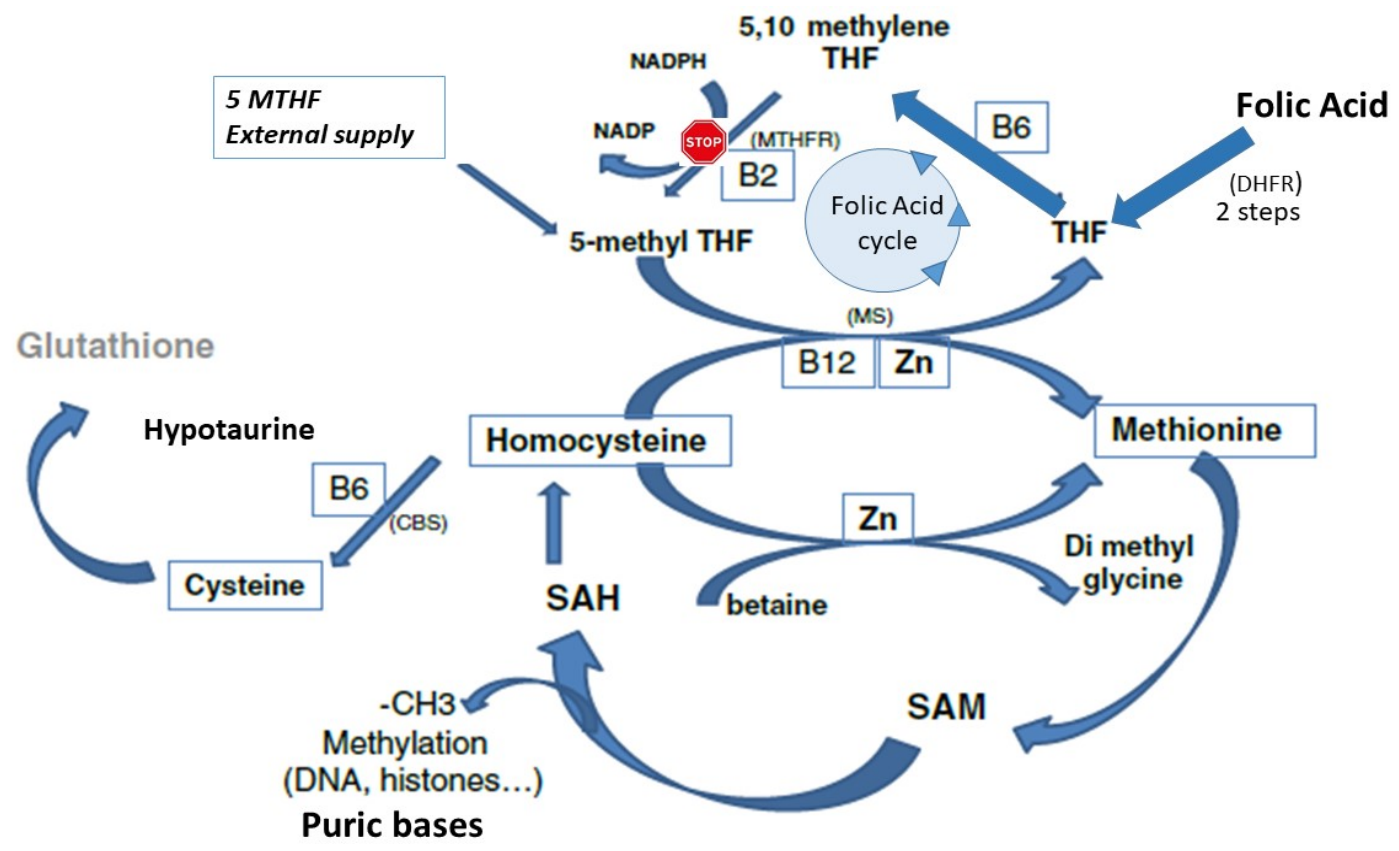

Figure 1. Folic acid and MTHFR.

Without a correct activity of MTHFR, folic acid intake is useless: It cannot feed the 1-CC (One carbon cycle) CBS: Cystathionine betasynthase pathway, DHFR: Di HydroFolate Reductase, MTHFR: Methylene Tetra Hydrofolate Reductase, MS: Methionine Synthase, SAH: S Adenosyl Homocysteine, SAM: S Adenosyl Methionine. Zn: Zinc. B2, B6, B12: vitamins B2, B6, B12.

5 MethylTHF: 5 Methyl Tetra Hydrofolate. It is the natural biochemically active Folate .

Table 1. Results of numerous studies conducted worldwide looking at the C677T mutation.

\begin{tabular}{|c|c|c|}
\hline Ethnic group & Carrier & Affected \\
\hline Hispanic American & $48 \%$ & $25 \%$ \\
\hline Caucasian American & $45 \%$ & $12 \%$ \\
\hline Japanese & $45 \%$ & $12 \%$ \\
\hline German & $37 \%$ & $6 \%$ \\
\hline Asian & $29 \%$ & $3 \%$ \\
\hline African American & $24 \%$ & $2 \%$ \\
\hline Sub-Saharan African & $12 \%$ & $1 \%$ \\
\hline
\end{tabular}

aspect is DNA and histone methylation: it is strongly involved in the process of epigenesis and imprinting. DNA methylation, after erasure during early embryo development, is restored during prenatal life in males, and during post-natal follicle development in females. Germcell-specific methylation and genomic imprints are established via de novo DNA methylation during oogenesis and spermatogenesis, through DNA methyltransferase(s) activity.

The universal cofactor for methylation is S-Adenosyl methionine (SAM). Normally, the oocyte and early embryo have a full capacity to synthesize SAM [6] and all intermediary metabolites involved in the methylation process. In all the steps, enzymes involved are expressed; but this capacity is decreasing with maternal age [7]. Once the target molecule has been methylated, S-Adenosyl homocysteine (SAH) is formed (Figure 1) and homocysteine (Hcy) is released after hydrolysis. Hcy is a toxic compound that must be recycled in Methionine via the One Carbon Cycle (1-CC); but the efficacy of the 1-CC relies strongly on the folic acid cycle.

In order to be metabolically active, folic acid must be converted to dihydrofolate and then to Tetrahydrofolate (THF) by the Dihydrofolate reductase (DHFR), see figure 1. Then THF can be transformed to the biologically active L-methyl folate by the enzyme methylenetetrahydrofolate reductase (MTHFR) and enter the 1-CC, to allow the conversion of Homocysteine to Methionine. MTHFR activity is a critical step. Even if some data in literature are confusing $[8,9]$, there are now strong evidences indicating that MTHFR isoforms, especially C677T are noxious for fertility in men [10] and women (11). C677T isoform severely alters preimplantation development and increases chromosomal abnormalities. It has also been associated with repeat early fetal loss or habitual first trimester abortions.

Methylation is fundamental to the proper function of almost all body's systems such as: repairing and regenerating cells, tissues and DNA; regulating gene expression and protein function; sensitizing neuro-transmitters (dopamine, epinephrine, norepinephrine, serotonin) that influence mood, sleep, behavior, cognition and memory; controlling homocysteine, an amino acid that can damage blood vessels; keeping inflammation in check; assisting the liver in processing fat, activating and regulating the immune system; modifying toxins and heavy metals. It also plays a role in detoxification of a variety of compounds, including toxins such as mercury, lead, arsenic and certain medications. Methyl folate, the metabolite of folic acid, is a key player in the methylation process.

\section{Folic Acid vs 5MTHF}

Folic acid is a synthetic folate form enjoying good stability and cheap manufacturing but it is very rare in nature and does not naturally occur in humans. It is used in the US in most vitamin supplements and extensively in fortified food. For example, all flour is systematically fortified with $140 \mu \mathrm{g} / 100 \mathrm{~g}$, so are pasta and cornmeal products. It is transformed into 5-methyl THF with the help of an intact MTHFR, B2 and B3 vitamins. 5-MTHF is the metabolically active form of folic acid obtained through a reductive process. 5-MTHF can like folic acid be formed. The best natural folate sources in foods are green, leafy vegetables. Cooking or processing destroys these compounds. Lentils, chickpeas, pinto beans, black-eye peas, avocados, broccolis, sprouts, fruits, brewer's yeast, liver and kidney also contain high amounts of folate [12]. 
Servy E (2017) The Methylene Tetrahydrofolate Reductase (MTHFR) isoform challenge. High doses of folic acid are not a suitable option compared to 5 Methyltetrahydrofolate treatment

The traditional recommendation for MTHFR gene mutation is folic acid supplement often prescribed in large quantity (400 to $800 \mu \mathrm{g}$ daily). Logically thinking, it would correct a folate deficiency. It has been shown recently that folic acid may not be a suitable solution.

\section{Unmetabolized folic acid (UMFA)}

Folic acid is metabolized in the liver and once the liver is saturated there is a significant amount of unmetabolized folic acid (UMFA) circulating freely in the blood. A dose of about 100 to $200 \mu \mathrm{g}$ is known to unlikely cause the appearance of UMFA in serum, but it is detected after a supplementation period of 14 weeks of $400 \mu \mathrm{g}$ that obviously leads to UMFA appearance in serum [13].

Also UMFA can be a problem for patients being treated with antifolate drugs for different diseases such as arthritis, psoriasis, malaria, leukemia and other cancers [14]. The antifolate drugs work by inhibiting dihydrofolate reductase. The most common drugs are Methotrexate (antineoplastic and immunosuppressant), Pemetrexed (antineoplastic), Proguanil (antimalarial), Pyrimethamine (antiprotozoal) and Trimethoprim (broad-spectrum antimicrobial). Correspondingly, high folic acid intake causes a reduction in natural killer cells (NK) decreasing the natural cytotoxicity immune function in response to tumor formation [15]. Interestingly, it is also well known that high folic consumption can block the binding of folate to all folate receptors leading to a pseudo MTHFR deficiency [16]. A large scale clinical trial regarding colorectal adenomas and prostate cancers revealed that not only folic acid supplement failed to prevent recurrence but caused a flare up of the tumoral process [17].

\section{Hyperhomocysteinemia}

Deficit in MTHFR activity may cause hyperhomocysteinemia and homocysteinuria with all their clinical manifestations including developmental delay, osteoporosis, thromboembolic and Cardiac diseases and premature atherosclerosis. It is associated with an increased risk of fetal open neural tubal defects. Serum homocysteine should be checked when there is MTHFR gene mutation, especially when dealing with a homozygous or compound heterozygous type. Homocysteine levels are typically higher in men than in women, and increase with age. Elevated levels if $>10.4 \mu \mathrm{mol} / \mathrm{L}$ or $>140 \mu \mathrm{g} / \mathrm{dL}$ (female) and if $>11.4$ $\mu \mathrm{mol} / \mathrm{L}$ or $>150 \mu \mathrm{g} / \mathrm{dL}$ (male). Hyperhomocysteinemia may also occur due to mutations in enzymes other than MTHFR or due to acquired factors.

For patients affected with MTHFR gene mutation, excess folic acid intake is not advised and can cause toxicity due the presence of UMFA. It leads to decrease via a negative competition, binding of the (natural) $5 \mathrm{MTHF}$ receptors and transporters. These individuals lack the enzyme that metabolizes folic acid to its active form. Therefore, the active form (5-MTHF) must be given, bypassing the defective enzyme and providing the body with the final active product, as well as reducing the homocysteine levels [18]. Individuals affected with MTHFR gene mutation must firstly modify their diet and decrease their intake of fortified wheat flour and folic acid supplements. Homozygous or compound heterozygous women who have experienced repeat fetal loss should be treated with active 5-MTHF (L-methylfolate) as well as taking low molecular weight heparin (LMWH) to counteract hypercoagulability due to potential hyperhomocysteinemia.

\section{Pathology concerning gametes and embryos}

MTHFR isoform has a real negative impact on early embryonic development, directly driven by the maternal stores or reserves previously accumulated. The negative impact is also observed on the male gamete. Folate-receptor-1 and folate-transporter-member-1 are two of the most expressed mRNAs in the oocyte [19]. Only portion of the ingested folates can be metabolized in carriers of MTHFR mutations and be used in the one-carbon-cycle (1-CC). As mentioned earlier, excess of folic acid can negatively compete for the absorption and binding on the receptor and transporter, with the natural Methylfolate ingested from food; therefore, it may aggravate the negative impact. Excess of folic acid leads to UMFA syndrome. 5-MTHF is more effective in lowering serum homocysteine. When comparing treatment with FA, 5-MTHF induces significantly higher plasma folate concentration. UMFA in plasma appears regularly after FA supplementation but rarely after 5-MTHF supplementation [20]. In addition, 5-MTHF increases plasma folate more effectively than FA irrespective of mutations of MTHFR.

\section{Habitual abortions}

In patients with repeat miscarriages and ART failures, a strong impact of the C677T MTHFR isoform is observed. Both partners could be responsible for the failure, not only the woman. MTHFR gene mutation has been recognized for many years as being one of the possible causes of repeat first trimester fetal losses .Hypercoagulability being linked to hyperhomocysteinemia, the patients are treated with anticoagulants to reduce the risk of thrombotic events in addition to $800 \mu \mathrm{g}$ of 5-MTHF. Low molecular weight heparin (LMWH) is administered starting at the beginning of the pregnancy [21].

\section{Assisted reproductive failures}

In patients with repeat losses and ART failures, a strong impact of the C677T MTHFR isoform has been observed. Oocyte donation failures are generally attributed in part to the male partner. It is imperative to address a question regarding oocyte and sperm donors, since MTHFR testing is not usually obtained in the screening of donors by banks storing and providing gametes. Also, testing should be mandatory in couples with repeat miscarriages ART failures. Testing the donor, the recipient and the male partner should be considered as proper medical practice. Couples with repeat intra uterine insemination (IUI) or in-vitro-fertilization (IVF) failures should be tested for MTHFR mutations. If MTHFR mutation is detected, the patients should be treated with $800 \mu \mathrm{g}$ of 5-MTHF with vitamin complements for 2 to 4 months before any further attempts. Successful experiences with such treatments have been reported [22].

\section{Abnormal semen analysis}

A negative impact of MTHFR C677T isoform on semen quality has been reported $[10,22]$ and $5 \mathrm{MTHF}$ allows a fertility improvement [22]. It may concern sperm morphology, oligo-asthenospermia and DNA tertiary structure. The paternal effect on embryo development should not be overlooked as trophoblast growth and differentiation are strongly under paternal control and request a high methylation activity. Testing sperm donors in cryopreservation banks should also be advocated.

\section{Premature ovarian insufficiency}

It has also been speculated that some cases of early ovarian failures could be attributed to an abnormal cellular methylation process [22]. Testing include a serum AMH (anti-mullerian hormone), a pelvic ultrasound with evaluation of the ovarian volume and number of antral follicles. All patients who are affected with a prematurely low ovarian reserve should be tested for a possible MTHFR mutation. 
Servy E (2017) The Methylene Tetrahydrofolate Reductase (MTHFR) isoform challenge. High doses of folic acid are not a suitable option compared to 5 Methyltetrahydrofolate treatment

\section{Prevention of neural tube defects}

A protective effect of folate against the development of neural tube defects (NTDs), specifically, anencephaly and spina bifida, has been well recognized for the past half century. All women capable of becoming pregnant have been urged to ingest folic acid regularly. The recommended intake was $4 \mathrm{mg}$ or $4,000 \mu \mathrm{g}$ per day for those at high risk (by virtue of a previous NTD pregnancy outcome) and $400 \mu \mathrm{g} / \mathrm{d}$ for all others. Folic acid fortification was mandated in the United States and some other countries. The process was followed by significant improvement in folate indexes and a reduction of $25-30 \%$ in NTD frequency (about one-half of the proportion of cases assumed to be responsive to folate). Nevertheless, several research gaps remain: identification of the mechanism by which the defect occurs and how folate ameliorates it $[2,4,23]$. In view of our present knowledge regarding the methylation process, women will be better served by taking $800 \mu \mathrm{g}$ of 5-MTHF than the previously advocated large doses of folic acid.

\section{Treatments: case series}

It has been shown that with equal doses of $100 \mu \mathrm{g}$, there is no or little difference between 5-MTHF and folic acid in generating plasma folate. In homozygous MTHFR mutation, the effects of treatments with 5 -MTHF $(400 \mu \mathrm{g})$ or folic acid $(400 \mu \mathrm{g})$ were compared. Results show that 5-MTHF induces significantly higher plasma folate concentration compared to folic acid. Furthermore, unmetabolized folic acid in plasma increases regularly after folic acid supplementation but appears rarely after 5-MTHF. The same report suggests that 5-MTHF increases plasma folate more effectively than folic acid irrespective of mutations of MTHFR [8,12].

In a first group of 7 couples having had between 5 to 9 miscarriages, a treatment with high doses of folic acid ( $5 \mathrm{mg} /$ days) have been totally inefficient. Two of the couples were recommended to participate in oocyte donation programs In 5 couples of this group, both members were C677T carriers; The patients (men and women) were treated for 4 months with 5 MTHF with support of the 1 carbon cycle (1-CC): Impryl (Pathenogen, Lugano, Switzerland or Tetrafolic, Nurilia Lyon France). Three spontaneous pregnancies and deliveries ( 3 baby girls) were achieved, one patient is now 25 weeks pregnant and one is 16 weeks pregnant. In the last two couples the woman was homozygous one with C1298C and one with T677T: for this last couple, the man was wild type (WT) and a pregnancy is starting, post treatment.

In a second group of 3 couples with 2, 2 and 3 oocyte donation failures, we observed that all the partners were affected by the C677T isoform, either homozygous or heterozygous. We made the same observation in another group of 3 couples with a heavy background of repeated IVF/ICSI failures. These 6 couples are currently under treatment. A complementary support of the 1-CC is essential: The onecarbon or methylation cycle needs a robust support and activation of the antioxidant system in order to complete the effect of 5-MTHF. The list of dietary useful to sustain the cycle is quite short and simple. There is need for B-complex vitamin containing quantities of riboflavin (B2), pyridoxin (B6), niacin (B3), cobalamin (B12) in order to facilitate the completion of the folic acid cycle. Vitamin B12 needs to be monitored to ensure that levels are adequate, to prevent the masking of B12 deficiency, which may lead to neuropathy. The presence of chelated zinc is also indispensable for the conversion of homocysteine into methionine. It is to be noted that only chelated zinc is actually bioavailable and that most commercial supplements contain zinc oxide that provides a negligible support [24]. It has to be mentioned that some other deficiencies in other enzymes of the 1-CC can be associated with the MTHFR isoforms [24]. Consideration should as well be given to the administration of aspirin or low molecular weight heparin, depending on the clinical situation, to prevent thrombotic events.

\section{Conclusions}

We should restrain from adding folic acid in the general population without MTHFR mutation. When administering folic acid, it is important to take into consideration the individuals intake of fortified food or supplements. A total dose of only $200 \mu \mathrm{g}$ or less should be safe. 5-MTHF is a better alternative because it's a natural form of form of folate directly available for gastrointestinal absorption. It is not affected by genetic metabolic defects and no upper intake limit. Also, it prevents the formation and excess of UMFA in circulating blood. Finally, 5 -MTHF is definitely indicated at a therapeutic dose of $800 \mu \mathrm{g}$ a day for all deficiencies due to MTHFR mutations. In this specific case, folic acid supplementation is not a suitable solution. In addition, all patients with repeat IVF failures or miscarriages must be tested for MTHFR isoforms. Failure to do so could now be qualified as poor practice. In light of the above findings we can suspect that there will be an increase in deliveries of progenies with a high rate of congenitally inherited MTHFR isoforms. Guidelines should be established for prophylaxis and treatment of children, especially when serum homocysteine is elevated [19].

\section{Conflict of interest}

The authors declare no conflict of interest.

\section{References}

1. Roy M, Leclerc D, Wu Q, Gupta S, Kruger WD, et al. (2008) Valproic acid increases expression of MTHFR and induces lower teratogenicityin MTHFR deficiency. $J$ Cell Biochem 105: 467-476. [Crossref]

2. van der Put NM, Gabreëls F, Stevens EM, Smeitink JA, Trijbels FJ, et al. (1998) A second common mutation in the methylenetetrahydrofolate reductase gene: an additional risk factor for neural-tube defects? Am J Hum Genet 62: 1044-1051. [Crossref]

3. National Center for Biotechnology Information (2016) Comparison of the Effect of Low-dose Supplementation with L-5-methyltetrahydrofolate or Folic Acid on Plasma Homocysteine. U.S National Library of Medicine.

4. Botto LD, Yang Q (2000) 5,10-Methylenetetrahydrofolate reductase gene variants and congenital anomalies: a HuGE review. Am J Epidemiol 151: 862-77. [Crossref]

5. Counsyl. (2014). MTHFR Deficiency. https://www.counsyl.com/services/family-prepscreen/diseases/mthfr-deficiency/

6. Menezo Y, Lichtblau I, Elder K (2013) New insights into human pre-implantation metabolism in vivo and in vitro. Assist Reprod Genet 30: 293-303. [Crossref]

7. Richardson B (2003) Impact on aging on DNA Methylation. Ageing Res Rev 2: 245 261. [Crossref]

8. Venn BJ, Green TJ, Moser R, Mann JI (2003) Comparison of the effect of low-dose supplementation with L-5-methyltetrahydrofolate or folic acid on plasma homocysteine: a randomized placebo-controlled study. Am J Clin Nutr 77: 658-662. [Crossref]

9. D'Elia PQ, Dos Santos AA, Bianco B, Barbosa CP, Christofolini DM (2014) MTHFR Polymorphisms C677T and A1298C and associations with IVF outcomes in Brazilian women. Reprod Biomed Online 28: 733-738. [Crossref]

10. Cornet D, Cohen M, Clement A, Amar E, Fournols L (2017) Association between the MTHFR-C677T isoform and structure of sperm DNA. J Assist Reprod Genet 34: 1283 1288. [Crossref]

11. Enciso M, Sarasa J, Xanthopoulou L, Bristow S, Bowles M, et al. (2016) Polymorphisms in the MTHFR gene influence embryo viability and the incidence of aneuploidy. Hum Genet 135: 555-568. [Crossref]

12. Carolyn Ledowsky, (2017) The folic acid vs 5-MTHF Debate. MTHFR Support Australia. https://www.mthfrsupport.com.au/folic-acid-vs-5-mthf-debate/

13. Sweeney MR, McPartlin J, Scott J (2007) Folic acid fortification and public health Report on threshold doses above which unmetabolised folic acid appear in serum. $B M C$ Public Health 7:41. [Crossref]

14. Kim YL (2008) Folic acid supplementation and cancer risk: point. Cancer Epidemiol Biomarkers Prev 17: 2220-2225. [Crossref] 
Servy E (2017) The Methylene Tetrahydrofolate Reductase (MTHFR) isoform challenge. High doses of folic acid are not a suitable option compared to 5 Methyltetrahydrofolate treatment

15. Sawaengsri H, Wang J, Reginaldo C, Steluti J, Wu D, et al. (2016) High folic acid intake reduces natural killer cell cytotoxicity in aged mice. $J$ Nutr Biochem 30: 102-107. [Crossref]

16. Xia W, Hilgenbrik AR, Matteson EL, Lockwood MB, Cheng JX, et al. (2009) A functional folate receptor is induced during macrophage activatio and can be used to target drugs to activated macrophages. Blood 113: 438-446. [Crossref]

17. Christensen KE, Mikael LG, Leung KY, Lévesque N, Deng L, et al. (2015). High folic acid consumption leads to pseudo-MTFHR deficiency, altered lipid metabolism, and liver injury in mice. Am J Clin Nutr 101: 646-658.

18. Lamers Y, Prinz-Langenohl R, Moser R, Pietrzik K (2004) Supplementation with 5-MTHF or folic acid equally reduces plasma total homocysteine concentrations. Am J Clin Nutr 79: 473-478. [Crossref]

19. Menezo Y, Servy E, Cohen M, Clement P (2017) Advanced Paternal age and Endocrine Disruptors: Two causes of Psychiatric Disorders in Children, with DNA Methylation Dys-Regulation as a Common Biochemical Mechanism. In Psychiatric Disorders www.smgebooks.com . Sept 20, 2017 pp 1-12.
20. Prinz-Langenohl R, Bramswig S, Tobolski, Smulders YM, Smith DE, et al. (2009) 5-MTHF increases plasma folate more effectively than folic acid in women with the homozygous or wild-type6777C ->T polymorphism of MTHFR. Br J Pharmacol 158: 2014-2021.

21. Deitcher S Hypercoagulable states (2010) www.clevelandclinicmeded.com/ medicalpubs/

22. Jacquesson-Fournols L, Servy EJ, Dale B, Clement A, Menezo Y (2017) MTHFR Testing in ART Should be Compulsory in both Male and Female Patients Including Oocyte Donation Programs. J Gynecol Women's Health 7: MS ID 555706.

23. Pitkin RM (2007) Folate and neural tube defects. Am J Clin Nutr 85: 285S-288S [Crossref]

24. Dattilo M, D'Amato G, Caroppo, Menezo Y (2016) Improvement of gamete quality by stimulating and feeding the endogenous antioxidant system: mechanisms, clinical results, insights on gene-environment interactions and the role of diet. J Assist Reprod Genet 33: 1633-1648. [Crossref]

Copyright: (C2017 Servy E. This is an open-access article distributed under the terms of the Creative Commons Attribution License, which permits unrestricted use, distribution, and reproduction in any medium, provided the original author and source are credited. 